Urologe 2010 · 49:9

DOI 10.1007/s00120-009-2206-y

C) Springer-Verlag 2010

\title{
Online-Einreichung von Manuskripten für Der Urologe
}

schen Einreichung, Begutachtung, eventueller Revision und schließlich Veröffentlichung.

- Ihre Daten sind sicher: Sie werden auf dem zentralen Datenserver gespeichert, der regelmäßig gesichert wird, sodass ein Datenverlust nicht vorkommt.

- Mehr Transparenz: Autoren können jederzeit online den aktuellen Stand der Begutachtung ihres eingereichten Artikels verfolgen.

Weitere Informationen zum Verfassen und Einreichen von Manuskripten finden Sie in der nebenstehenden Infobox.

\section{Ihre Schriftleitung DER UROLOGE}

\section{Für die Schriftleitung}

\section{Prof. Dr. H. Rübben}

Urologische Universitätsklinik,

Hufelandstr. 55, 45122 Essen

herbert.ruebben@uni-essen.de

\section{Manuskripte online einreichen}

Bitte folgen Sie auf der Homepage www.DerUrologe.de dem Link „Für Autoren“.

Klicken Sie bitte unter „Editorial Manager" auf "Manuskript online einreichen". Hier finden Sie auch die Autorenleitfäden und Musterbeiträge mit Hinweisen für die Manuskriptgestaltung.

Bei der ersten Nutzung registrieren Sie sich unter „Register" mit der Angabe Ihrer persönlichen Daten und eines User-Namen. Ein Passwort wird Ihnen nach Registrierung per E-mail automatisch zugeschickt. Mit lhrem User-Namen und Passwort haben Sie als Autor zukünftig direkten Zugang zum System. Nach der Anmeldung (Login) als Autor können Sie nun Ihren Beitrag online einreichen.

Sollten Sie noch Fragen zur Manuskripteinreichung haben, wenden Sie sich bitte an die Redaktion beim Herausgeber:

\section{Dr. Frank vom Dorp}

Redaktion Editorial Manager

der Zeitschrift DER UROLOGE

Tel. 0201/723-3210

Frank.vomDorp@uk-essen.de

\section{www.DerUrologe.de}

oder direkt zu

www.editorialmanager.com/deur nicht mehr nötig: Die Online-Einreichung bietet die automatische Datenkonversion der Urdatei in eine PDFDatei - ein stabiles Dokument, das überall an jedem Computer gleich aussieht.

- Der Begutachtungsprozess wird beschleunigt: Das Redaktionssystem ermöglicht die nahtlose Verbindung zwi-

Die Online-Einreichung hat zahlreiche Vorteile:

- Datenträger oder Papierversionen sind

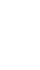

